\title{
Clicking cyclophane to boron doped diamond surfaces
}

\author{
WANG Mei \\ Key Laboratory of Liquid Structure and Heredity of Materials, Ministry of Education, Shandong University, Jinan 250061, China
}

Received July 13, 2012; accepted September 24, 2012; published online April 24, 2013

\begin{abstract}
"Click chemistry" was used to covalently attach acetylene-bearing cyclophane to azide-terminated BDD surfaces. The azide termination was obtained through an esterification reaction between 4-azidobenzoic acid and the terminal hydroxyl groups of oxidized BDD surfaces. The resulting surfaces were characterized using X-ray photoelectron spectroscopy (XPS), water contact angle and electrochemical measurements. As a result, alkyne-functionalized cyclophane can undergo click chemistry to conveniently attach these units onto a preformed azide-functionalized BDD surface.
\end{abstract}

cyclophane, boron doped diamond, click chemistry, esterification, azide

Citation: Wang M. Clicking cyclophane to boron doped diamond surfaces. Chin Sci Bull, 2013, 58: 2898-2902, doi: 10.1007/s11434-013-5703-8

The concept of click chemistry was introduced in 2002 by Sharpless [1]. It is based on the copper (I) catalyzed triazole formation through the classic Huisgen 1,3-dipolar cycloaddition between azides and alkynes [1,2]. It represents a chemically simple conjugation approach fulfilling many of the crucial requirements for mild surface immobilization $[3,4]$. Recently, these "click" reactions on polymer and solid surfaces have been reviewed [5,6], and due to its high selectivity, high yields and mild reaction conditions, numerous strategies to exploit this reaction to are now attractive to surface scientists [7,8]. Azides and alkynes are highly energetic functional groups with particularly narrow distributions of reactivity. Thanks to their weak acid-base properties, they are nearly inert toward biological molecules and the reaction conditions found inside living cells. Furthermore, azide and alkyne groups are easy to introduce into organic compounds by both nucleophilic and electrophilic reactions. One may therefore envision their incorporation into biological molecules by organic synthesis and chemical conjugation. Recently, this "click" methodology has been extended to feature interlocked structures (e.g. rotaxanes and catenanes) [9-16]. This methodology offers higher yields and better resulting structure compared to traditional methods.

email: wangmei@sdu.edu.cn
In the search for new strategies to derivatize BDD surfaces, we extend this "click" methodology to a new alkynefunctionalized cyclophane conveniently "clicked" onto an azide-functionalized boron doped diamond surface, as shown in Figure 1. The synthesis of the alkyne-functionalized cyclophane was described in the literature [11]. Azide termination was obtained by esterification reaction of surface hydroxyl groups of the oxidized BDD with 4-azidobenzoic acid at room temperature, as shown in Figure 2. The advantage of using 4-azidobenzoic acid, a commercially available molecule, for the introduction of an azide group on the BDD surface is the fact that this molecule can be easily coupled to any $-\mathrm{OH},-\mathrm{NH}_{2}$ or $-\mathrm{SH}$ terminated surface.

\section{Experimental}

\subsection{Materials}

All cleaning reagents were clean-room grade. All chemicals were reagent grade or higher and were used as received unless otherwise specified. Milli-Q water was used for all experiments. $N, N$-Dicyclohexylcarbodiimide (DCC), 4-Dimethylaminopyridine (DMAP), CuI, Anhydrous tetrahydrofuran (THF), Anhydrous dimethylformamide (DMF) and tetrabutylammonium tetrafluoroborate $\left(\mathrm{Bu}_{4} \mathrm{NBF}_{4}\right)$ were purchased 


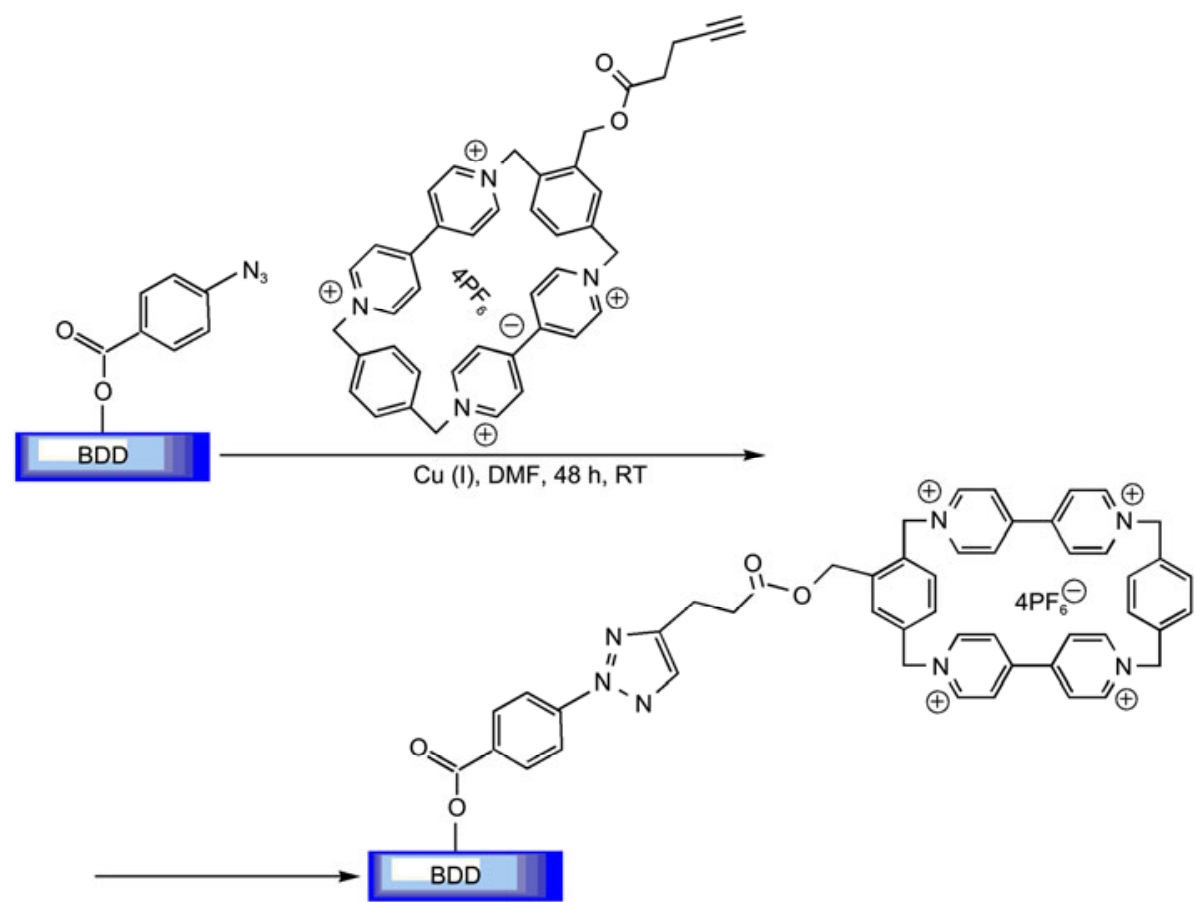

Figure 1 Scheme of clicking ethynyl cyclophane to azide-terminated diamond surface.

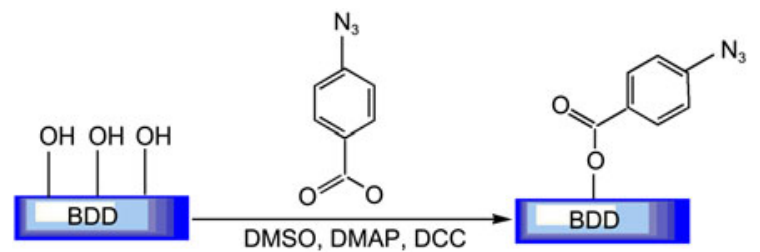

Figure 2 Esterification reaction scheme on diamond surface.

from Aldrich (Lyon, France). 4-Azidobenzoic acid was obtained from TCI Europe (Brussels, Belgium).

\subsection{BDD sample preparation}

Polycrystalline diamond layers were synthesized on a high purity p-type silicon wafer using microwave plasma-enhanced chemical vapor deposition (PECVD) technique in a conventional reactor [17]. The growth conditions used were as follows: substrate temperature $700-900^{\circ} \mathrm{C}$, total gas flow of a mixture of $0.7 \%$ methane in hydrogen $100 \mathrm{sccm}$; total pressure in the reactor 20 Torr (30 mbar); microwave power $700 \mathrm{~W}$. The dopant source was boron oxide set in a Pt crucible placed on the substrate holder near the silicon substrate.

\subsection{Photochemical oxidation}

A low pressure mercury arc lamp (UVO cleaner, Jelight, USA) was used to photochemically oxidize BDD samples for 55 mins as reported previously [18].

\subsection{Esterification}

4-Azidobenzoic acid (2 mmol), DCC (2.2 mmol) and $45 \mathrm{mg}$ of DMAP were dissolved in $20 \mathrm{~mL}$ dry THF. The oxidized BDD was immersed in the solution and left at room temperature for $24 \mathrm{~h}$ under a nitrogen atmosphere. The sample was removed from the solution, washed with THF, dichloromethane, ethanol and finally with water, and then dried under a nitrogen stream.

\section{5 "Clicking" cyclophane on azide-terminated BDD surface}

The azide-terminated BDD surface was immersed in a solution of alkyne-functionalized cyclophane $(0.04 \mathrm{mmol})$ in DMF $(10 \mathrm{~mL})$ at room temperature. Then $\mathrm{CuI}(0.004 \mathrm{mmol})$ was added. The solution was kept for $48 \mathrm{~h}$ in the dark in a glove box and the solvent was carefully removed under high vacuum. DMF was added to remove unreacted starting materials. The resulting surfaces were washed with THF and dried under a stream of nitrogen.

\subsection{Surface characterization}

Water contact angles were measured using deionized water. A DropMaster 500 (Kyowa Interface Science, Saitama, Japan) was used for measuring the contact angles. X-ray photoelectron spectroscopy (XPS) measurements were performed with an ESCALAB $220 \mathrm{XL}$ spectrometer from vacuum Generators. All electrochemical experiments were performed using an Autolab potentiostat 30 (Eco Chemie, Utrecht, and the Netherlands). The sample was mounted in a polytetrafluoroethylene (PTFE) holder with a copper contact. Electrochemical experiments were performed using a classical three-electrode device in a solution of acetonitrile $(0.1 \mathrm{~mol} / \mathrm{L}$ 
Bu4NPF6) with a platinum wire was used as counter electrode and BDD as working electrode.

\section{Results and discussion}

\subsection{Water contact angle measurements}

Water contact angle measurements were used to examine the macroscopic evolution in the wetting properties of the boron-doped diamond electrode before and after functionalization (Table 1).

The as-prepared BDD is hydrogen-terminated. This termination confers a hydrophobic character to the surface with a water contact angle $94 \pm 2^{\circ}$. Photochemical oxidation of the BDD substrate yields a surface termination with a hydrophilic character. The contact angle value decreased significantly to $8^{\circ}$. Chemical esterification of the terminal hydroxyl groups with 4-azidobenzoic acid led to an increase in contact angle. A value of $57 \pm 2^{\circ}$ was measured for the azide-terminated BDD surface. After clicking cyclophane groups to the azide terminated BDD surface, the contact angle dropped to $20 \pm 2^{\circ}$, as compared to $57 \pm 2^{\circ}$ for the azide termination surface (Table 1), indicating that the cyclophane groups display a hydrophilic character.

\subsection{X-ray photoelectron spectroscopy (XPS) analysis}

Figure 3(a) displays the XPS survey spectrum of the azide terminated BDD surface. It shows a main peak at $285 \mathrm{eV}$ due to $\mathrm{C} 1 \mathrm{~s}$ and a peak at $532 \mathrm{eV}$ due to $\mathrm{O} 1 \mathrm{~s}$. An additional peak at $\sim 400 \mathrm{eV}$, characteristic of nitrogen $(\mathrm{N} 1 \mathrm{~s})$ is also observed. High resolution XPS investigation of the $\mathrm{N} 1 \mathrm{~s}$ was performed (Figure 3(b)). Careful analysis showed two peaks at 401 and $406 \mathrm{eV}$, suggesting the presence of two nitrogen species in the azide-terminated monolayer, and reflects

Table 1 Contact angles of diamond surfaces in the course of clicking

\begin{tabular}{ccccc}
\hline & H-BDD & HO-BDD & $\mathrm{N}_{3}$-BDD & cyclophane-BDD \\
\hline Contact angle & $94 \pm 2^{\circ}$ & $8 \pm 2^{\circ}$ & $57 \pm 2^{\circ}$ & $20 \pm 2^{\circ}$ \\
\hline
\end{tabular}

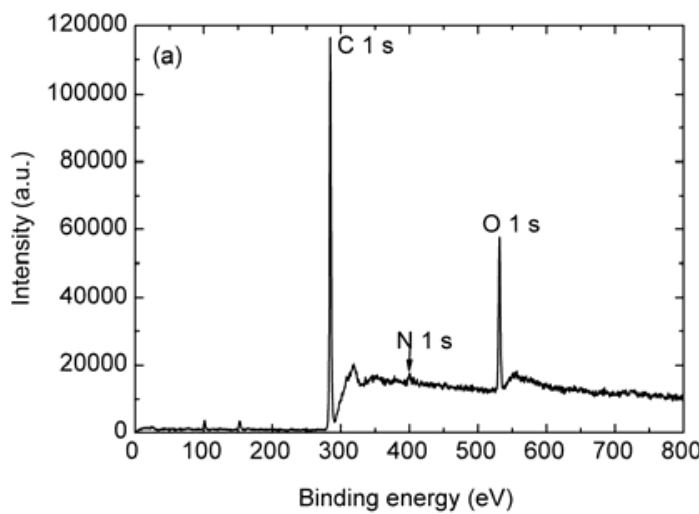

the differently charged nitrogen atoms in the azide group [19-21]. This indicates the success of the esterification reaction.

To investigate the changes in the surface composition after the introduction of the cyclophane functionality on the azide-terminated BDD surface, cyclophane-terminated BDD surface was also analyzed by XPS (Figure 4). XPS survey spectrum is shown in Figure 4(a). When compared with the XPS survey spectrum of the azide-terminated BDD surface (Figure 3), a new peak at $688 \mathrm{eV}$ ascribed to F1s appeared after clicking cyclophane groups onto the azide-terminated BDD surface. The high resolution XPS spectrum of F $1 \mathrm{~s}$ is displayed in Figure 4(b). It consists of a single peak at 688 $\mathrm{eV}$. Two expected peaks at 110 and $136 \mathrm{eV}$ due to phosphorus atom were obeserved as expected for the cyclophane moieties. Figure 4(c) displays the high resolution XPS spectrum of N1s of the cyclophane modified BDD surface, showing two peaks at 399.4 and $401.6 \mathrm{eV}$ corresponding to nitrogen in the form of 1,2,3-triazole and in the aromatic ring, respectively. All above indicates that cyclophane has been successfully clicked on the azide-terminated BDD surface.

\subsection{Electrochemistry measurement}

We have further exploited the electrochemical signature of the electroactive cyclophane moiety to investigate whether the cyclophane is grafted onto the BDD surface using click chemistry. The cyclic voltammogram of the cyclophaneterminated BDD surface recorded in acetonitrile $(0.1 \mathrm{~mol} / \mathrm{L}$ $\mathrm{Bu}_{4} \mathrm{NPF}_{6}$ ) (Figure 5) reveals the formation of two redox waves (absent in the azide terminated surface), presumably corresponding to the sequential formation of the diradical dication and fully reduced states of the cyclophane, respectively $[11,22]$. However, significant attenuation of the current was observed, which is consistent with surface inhibition due to the presence of either an overall low density surface grafting and/or the presence of defect domains. For further understanding, cyclic voltammograms of cyclophane solution on an azide modified BDD surface as well as on gold surface were investigated under the same conditions. Since two excellent redox waves were observed on gold surface, much

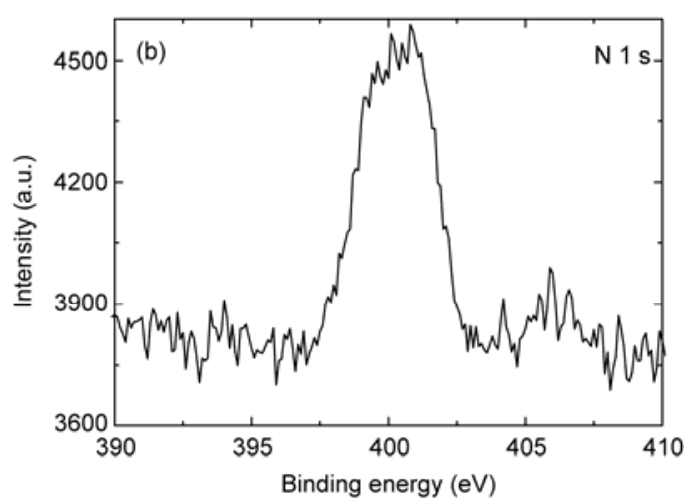

Figure 3 XPS survey spectrum of the azide-terminated BDD surface (a) and high-resolution XPS spectrum of the N $1 \mathrm{~s}$ peak (b). 

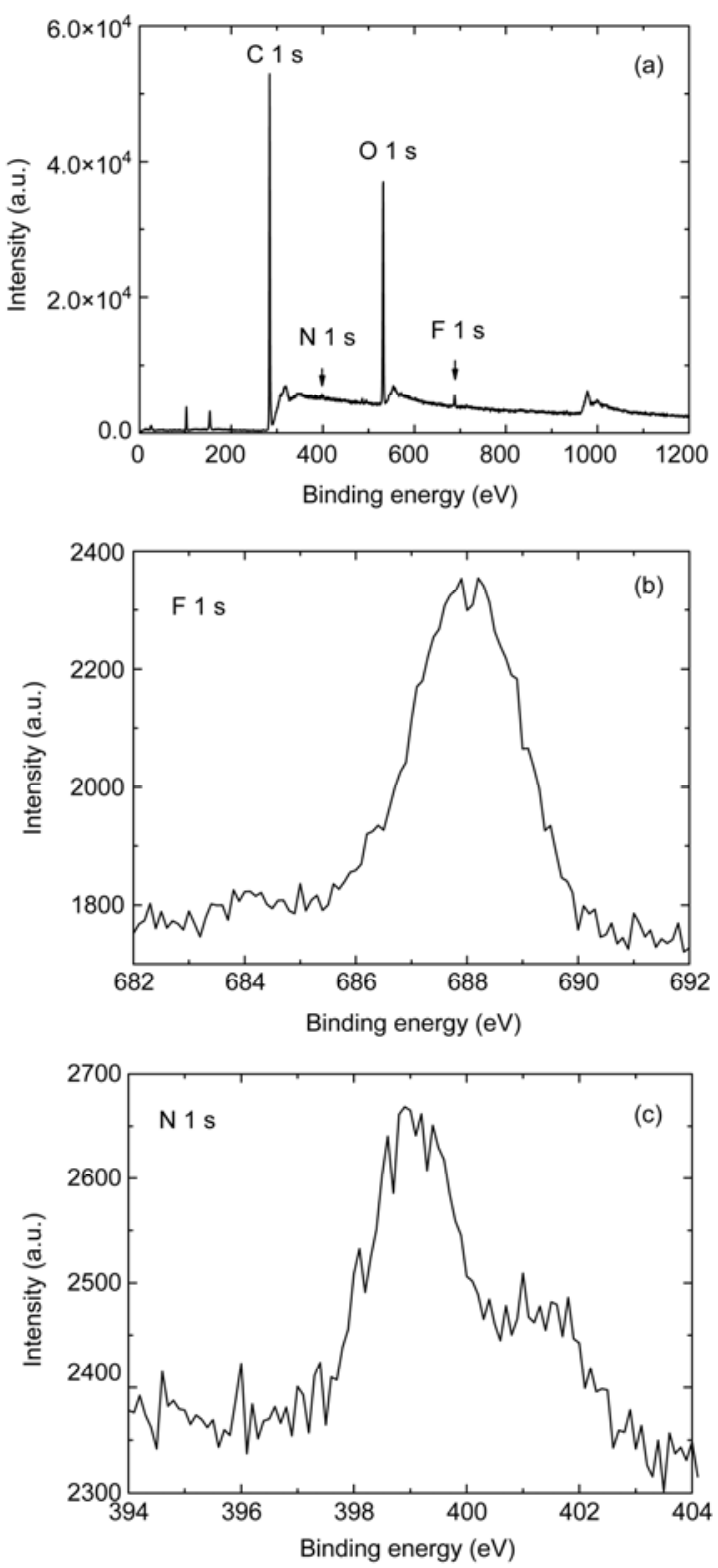

Figure 4 XPS survey spectra of cyclophane terminated BDD surface (a) highresolution XPS spectrum of F $1 \mathrm{~s}$ (b) high-resolution XPS spectrum of N $1 \mathrm{~s}$ (c).

lower current on the former surface indicates a more blocked surface. As a whole, cyclophane covalently linked on BDD surface was further confirmed.

\section{Conclusions}

In conclusion, we have demonstrated that "click" chemistry can be successfully applied for coupling functional cyclophane bearing a terminal acetylene group to azide-terminated BDD surfaces. Because of the gentle nature of the procedure, the strategy developed in this work can be used as a general platform to prepare functionalized surfaces for various applications. Based on the successful linking of cyclophane to the azide-terminated BDD surface, we are

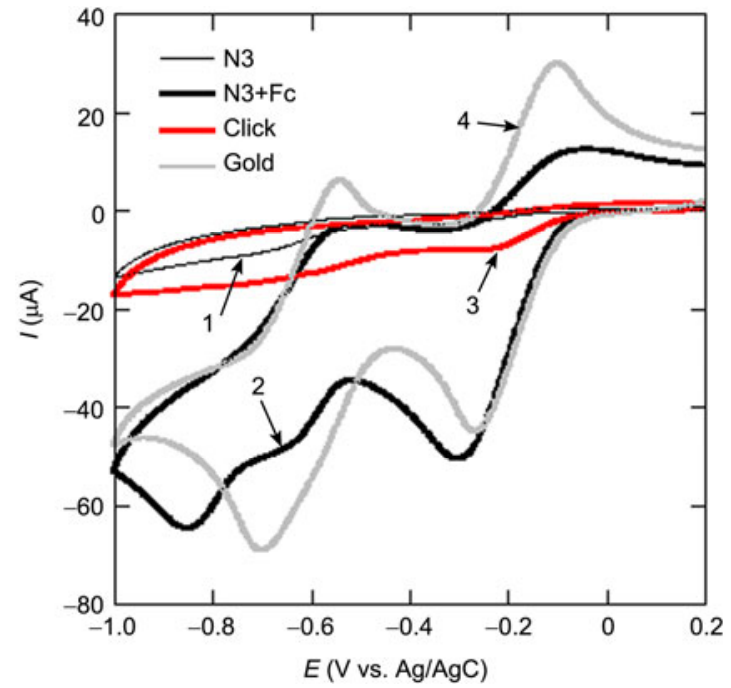

Figure 5 Cyclic voltammogram of azide modified BDD surface (curve 1), cyclophane solution on azide modified BDD surface (curve 2), cyclophane clicked BDD surface (curve 3), cyclophane solution on gold surface (curve $4)$ in acetonitrile $\left(0.1 \mathrm{~mol} / \mathrm{L} \mathrm{Bu}_{4} \mathrm{NPF}_{6}\right)$ solution. Scan rate: $0.1 \mathrm{~V} \mathrm{~s}^{-1}$.

currently exploiting the covalent coupling of the corresponding rotaxane and catenane onto azide terminated BDD surfaces using click chemistry. The applicability of this reaction scheme for coupling alkyne-terminated carbohydrates and other alkyne-functionalized molecules to flat supports is currently under investigation in our laboratory.

This work was supported by the National Natural Science Foundation of China (51002090) and the Outstanding Young Scientist Research Award Fund of Shandong Province (BS2010CL028).

1 Rostovtsev V V, Green L G, Fokin V V, et al. A stepwise huisgen cycloaddition process: Copper (I)-catalyzed regioselective ligation of azides and terminal alkynes. Angew Chem Int Ed, 2002, 41: 25962599

2 Tornoe C V, Christensen C, Meldal M. Peptidotriazoles on solid phase: [1,2,3]-triazoles by regiospecific copper (I)-catalyzed 1,3-dipolar cycloadditions of terminal alkynes to azides. J Org Chem, 2002, 67: 3057-3064

3 Kolb H C, Sharpless K B. The growing impact of click chemistry on drug discovery. Drug Disc Today, 2003, 8: 1128-1137

4 Binder W H, Sachsenhofer R. "Click" chemistry in polymer and materials science. Rapid Commun, 2007, 28: 15-54

5 Lutz J F. 1, 3-Dipolar cycloadditions of azides and alkynes: A universal ligation tool in polymer and materials science. Angew Chem Int Ed, 2007, 46: 1018-1025

6 Devaraj N K, Collman J P. Copper catalyzed azide-alkyne cycloadditions on solid surfaces: Applications and future directions. QSAR Comb Sci, 2007, 26: 1253-1260

7 Ciampi S, Böcking T, Kilian K A, et al. Functionalization of acetylene-terminated monolayers on Si (100) Surfaces: A Click Chemistry Approach. Langmuir, 2007, 23: 9320-9329

8 Marrani A G, Dalchiele E A, Zanoni R, et al. Functionalization of Si (100) with Ferrocene Derivatives via "Click" chemistry. Electrochim Acta, 2008, 53: 3903-3909

9 Miljanic O S, Dichtel W R, Mortezaei S, et al. Org Lett, 2006, 8: 4835-4838

10 Dichtel W R, Miljanic O S, Spruell J M, et al. Efficient templated 
synthesis of donor-acceptor rotaxanes using click chemistry. J Am Chem Soc, 2006, 128: 10388-10390

11 Mobian P, Collin J P, Sauvage J P. Efficient synthesis of a labile copper (I)-rotaxane complex using click chemistry. Tetrahedron Lett, 2006, 47: 4907-4909

12 Aucagne V, Haenni K D, Leigh D A, et al. Catalytic "click" rotaxanes: A substoichiometric metal-template pathway to mechanically interlocked architectures. J Am Chem Soc, 2006, 128: 2186-2187

13 Tuncel D, Steinke J H G. Catalytic self-threading: A new route for the synthesis of polyrotaxanes. Macromolecules, 2004, 37: 288-302

14 Aprahamian I, Dichtel W R, Iked T A, et al. A Clicked bistable [2]rotaxane. Org Lett, 2007, 9: 1287-1290

15 Braunschweig A B, Dichtel W R, Miljanic O S, et al. Modular synthesis and dynamics of a variety of donor-acceptor interlocked compounds prepared by click chemistry. Chem. An Asian J, 2007, 2: 634-637

16 Spruell J M, Dichtel M R, Heath J R, et al. The power of click chemistry for molecular machines and surface patterning. Chem A Eur J,
2008, 14: 4168-4177

17 Mermoux M, Fayette L, Marcus B, et al. In situ Raman monitoring of the growth of diamond films in plasma-assisted CVD reactors. Diam Relat Mater, 1995, 4: 745-749

18 Wang M, Simon N, Charrier G, et al. Distinction between surface hydroxyl and ether groups on boron-doped diamond electrodes using a chemical approach. Electrochem Commun, 2010, 12: 351-354

19 Lee M T, Ferguson G S. Stepwise Synthesis of a well-defined silicon (oxide)/polyimide interface. Langmuir, 2001, 17: 762-767

20 AlBataineh S A, Britcher L G, Griesser H J. XPS characterization of the surface immobilization of antibacterial furanones. Surf Sci, 2006, 600: 952-962

21 Haensch C, Hoeppene S, Schubert U S. Chemical surface reactions by click chemistry: Coumarin dye modification of 11-bromoundecyltrichlorosilane monolayers. Nanotechnology, 2008, 19: 035703-035710

22 Anelli P L, Ashton P R, Ballardini R, et al. Molecular meccano. 1. [2]rotaxanes and a [2]catenane made to order. J Am Chem Soc, 1992, 114: $193-218$

Open Access This article is distributed under the terms of the Creative Commons Attribution License which permits any use, distribution, and reproduction in any medium, provided the original author(s) and source are credited. 